\title{
Cloning and Characterization of Sdga Gene Encoding $\alpha$-Subunit of Heterotrimeric Guanosine 5'-Triphosphate-Binding Protein Complex in Scoparia dulcis
}

\author{
Masato Shite, Yoshimi Yamamura, Toshimitsu Hayashi, and Fumiya Kurosaki* \\ Graduate School of Medicine and Pharmaceutical Science for Research, University of Toyama; Sugitani 2630, Toyama \\ 930-0194, Japan. Received May 9, 2008; accepted July 23, 2008; published online August 27, 2008
}

\begin{abstract}
A homology-based cloning strategy yielded $S d g a$, a cDNA clone presumably encoding $\alpha$-subunit of heterotrimeric guanosine $5^{\prime}$-triphosphate-binding protein complex, from leaf tissues of Scoparia dulcis. Phylogenetic tree analysis of G-protein $\alpha$-subunits from various biological sources suggested that, unlike in animal cells, classification of $\mathrm{G} \alpha$-proteins into specific subfamilies could not be applicable to the proteins from higher plants. Restriction digests of genomic DNA of $S$. dulcis showed a single hybridized signal in Southern blot analysis, suggesting that $S d g a$ is a sole gene encoding $G \alpha$-subunit in this plant. The expression level of $S d g a$ appeared to be maintained at almost constant level after exposure of the leaves to methyl jasmonate as analyzed by reverse-transcription polymerase chain reaction. These results suggest that $S d g a$ plays roles in methyl jasmonate-induced responses of $\boldsymbol{S}$. dulcis without a notable change in the transcriptional level.
\end{abstract}

Key words $\alpha$-subunit; guanosine $5^{\prime}$-triphosphate-binding protein; heterotrimer complex; Scoparia dulcis

Higher plants produce jasmonic acid and its esters such as methyl jasmonate (MJ) in response to many stresses, and these compounds act as signaling messengers for the induction of defensive reactions involving phytoalexin production. $^{1-3)}$ Recent studies have shown that the activation of secondary metabolism in plants by jasmonates is not limited to the inducible defense-related compounds, but the biosynthesis of constitutively produced secondary metabolites is also enhanced by the exogenous supply of these hormones. ${ }^{4}$ It has been recently shown that jasmonates-induced responses in plant cells are mediated by heterotrimeric guanosine $5^{\prime}$ triphosphate (GTP)-binding protein complex. ${ }^{5}$ As well as in mammalian cells, this protein complex has been shown to participate in a variety of cellular processes of plants, such as blue or red light-mediated responses, ${ }^{6}$ ) plant hormone signaling, ${ }^{7)}$ and pathogen-induced resistance mechanisms. ${ }^{8)}$ It is well known that $\alpha$-subunits of G-protein complex in mammalian cells are generally composed of more than 20 isoforms ${ }^{9,10)}$; however, in contrast, it has been demonstrated that $\mathrm{G} \alpha$ proteins in plants are usually encoded by only one or two genes in the genomes. ${ }^{11-15)}$

We showed previously ${ }^{16)}$ that the biosynthetic activity of a series of tetracyclic diterpenoids, such as scopadulcic acid, scopadulciol, and scopadulin, in Scoparia dulcis is markedly increased when the plant was treated with MJ. We have also demonstrated that the activation of Ca-cascade in S. dulcis is an essential event for MJ-induced enhancement of the diterpenoids production, and calmodulin (CAM), a well known Ca-modulator protein of eukaryote cells, functions as a key component in the elevation of expression levels of several genes encoding biosynthetic enzymes of theses compounds. ${ }^{17)}$ It was expected, therefore, that heterotrimer Gprotein complex and CAM would play important roles in MJ-induced activation of terpenoids biosynthesis in S. dulcis. Recent studies clearly showed that plant CAM proteins are usually composed of several isoforms, and they are encoded by $>10$ genes. ${ }^{18,19)}$ The specific expression of these CAM genes in plants is sometimes induced upon the contact of the cells with appropriate stimuli or under stress conditions.
However, we have recently found ${ }^{20)}$ that, unlike in many higher plants, CAM in $S$. dulcis is encoded by an only one gene in the genome.

The primary aim of the present study was the elucidation of genomic organization of gene(s) encoding $\alpha$-subunit of heterotrimeric G-protein in $S$. dulcis, a unique "sole CAM gene" plant. First, an attempt was made to isolate G $\alpha$-subunit gene(s) from $S$. dulcis, and Southern hybridization analysis was performed for the restriction digests of genomic DNA of the plant. Possible change in the expression level of the gene after the treatment with MJ was also examined.

\section{MATERIALS AND METHODS}

Cloning of Sdga Gene from $\boldsymbol{S}$. dulcis $S$. dulcis was grown in a green house of the Experimental Station for Medicinal Plant Research, University of Toyama, and total RNA was isolated from leaf tissues of the plant with RNeasy Plant Mini Kit (Qiagen). The RNA obtained (approximately $1 \mu \mathrm{g}$ ) was subjected to rapid amplification of cDNA ends (RACE) methods using the GeneRacer Kit (Invitrogen) after generation of cDNA templates by reverse-transcription (RT) reaction with AMV-RT. The 3'-RACE was performed with GeneRacer Oligo dT as the reverse primer, and $5^{\prime}$-TAC CCA CGT CTA ACT AGA GAT CTT GCT CG-3', 5'-GCT CAA ACT GAT GCA GAC TCC TCA-3' as the gene-specific forward primers for polymerase chain reaction (PCR) amplification of the DNA fragments. The 5'-RACE was carried out with GeneRacer RNA Oligo as the forward, and 5'-CAA CAC AAT CAG GAT TCG TGC TCT GG-3', 5' -CTC GAT ATC GCG AGC AAG ATC TCT AG-3' as the reverse primers. The DNA fragments thus obtained were subcloned into the pCR2.1-TOPO vector (Invitrogen), and the nucleotide sequences were determined on both strands using the dye-terminator method with M13-20 and RV-P (Takara) as the sequencing primers on a PRISM 3100 Genetic Analyzer (Applied Biosystems).

Southern Blot Hybridization of Sdga Gene Genomic DNA was isolated from leaves of $S$. dulcis using OmniPrep 
(G-Biosciences) according to the instruction manuals, and the restriction digests were prepared using $X b a \mathrm{I}, X h o \mathrm{I}$, $E c o$ RI, or EcoRV (Takara). They were electrophoresed on a $0.8 \%$ agarose gel, and the separated DNA fragments were transferred onto an Immobilon-NY ${ }^{+}$(Millipore). The translatable region of $S d g a$ including stop codon (1152 nucleotides) was amplified by PCR and was directly labeled with AlkPhos Direct Labeling and Detection System (GE Healthcare Bio-Science) to be used as the probe. The membrane was hybridized with the probe at $55^{\circ} \mathrm{C}$ for $24 \mathrm{~h}$ in a solution containing $6 \times \mathrm{SSC}$, and, after appropriate washings, the filters were dried and exposed to an X-ray film at room temperature for $3 \mathrm{~h}$.
Expression of Sdga Gene The expression level of $S d g a$ was analyzed semi-quantitatively using RT-PCR. The leaves of $S$. dulcis were detached from the middle part of the stem, and incubated with MJ $\left(100 \mu \mathrm{moll}^{-1}\right.$ water, Wako Pure Chemicals) in Petri dishes at $26^{\circ} \mathrm{C}$ under constant illumination according to the method described previously. ${ }^{20)}$ Control treatments received only water instead of MJ. At regular intervals, the treated leaves were harvested and total RNA was isolated according to the method described above. Aliquots of RNA solutions (approximately $0.5 \mu \mathrm{g}$ RNA equivalent) were subjected to RT reaction using Transcriptor First Strand cDNA Synthesis Kit (Roche), and then, PCR was carried out with the primer pair, 5'-ATG CGG TGG ATG CTA ATC
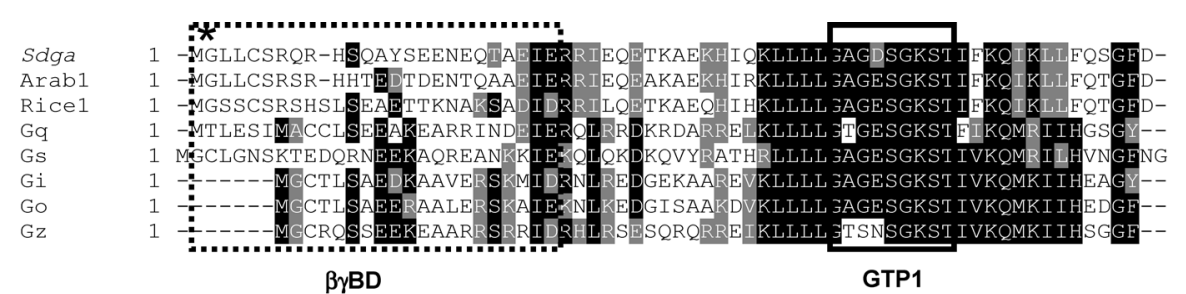

Sdga
Arab1
Rice1
Gq
Gs
Gi
Go
Gz
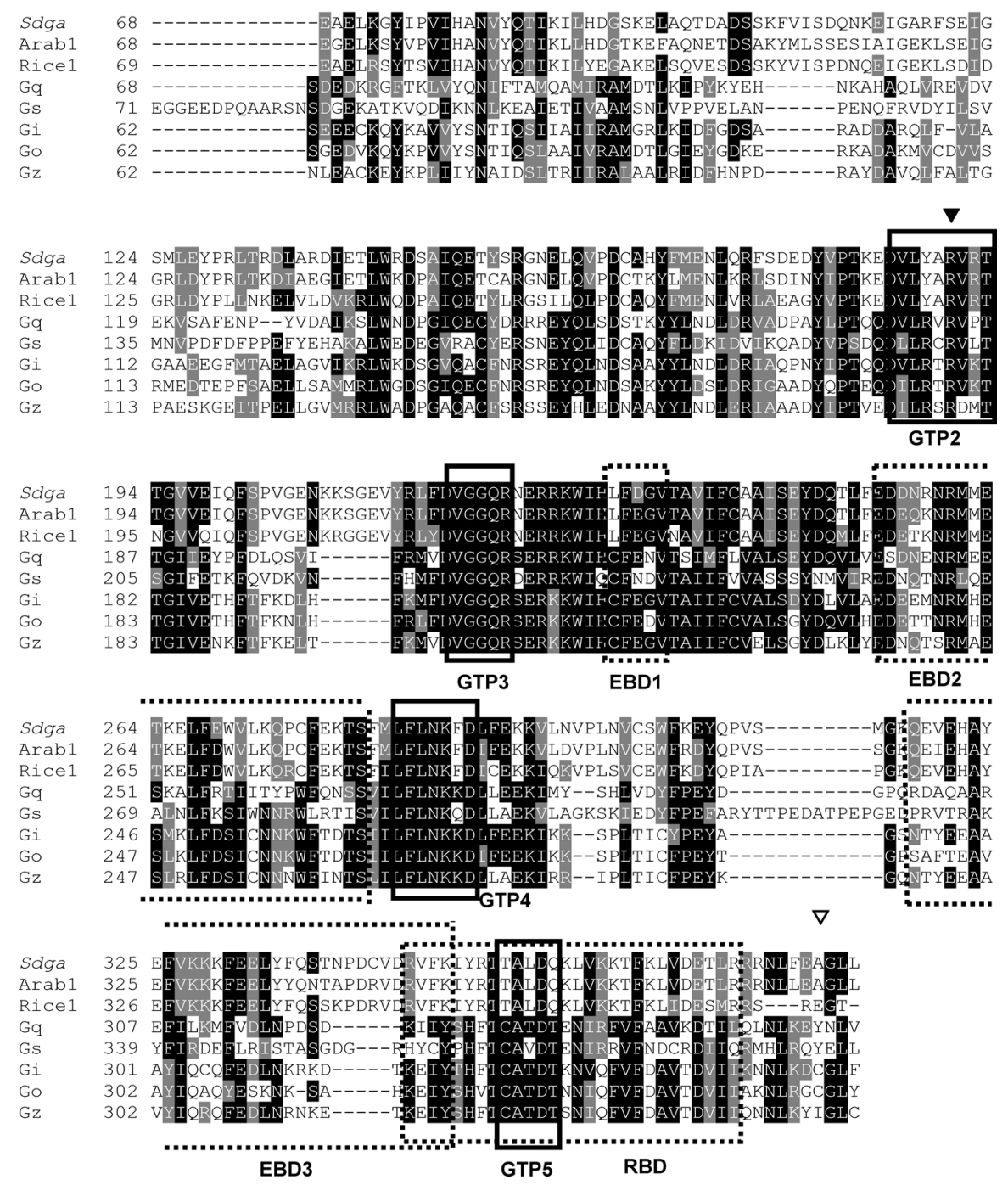

Fig. 1. Alignment of Amino Acids Sequences of $\alpha$-Subunits of GTP-Binding Protein Complex from Various Biological Sources

A cDNA clone encoding the $\alpha$-subunit of GTP-binding protein, Sdga, was isolated from $S$. dulcis, and the deduced amino acid sequence was compared with the proteins from various biological sources (Arab, Arabidopsis thaliana; Rice, Oryza sativa; Gq, Gs, Gi, Go and Gz are $\alpha$-subunit proteins from human). Their GenBank accession numbers are shown in Fig. 2, and identical and resembling amino acids are presented as black and gray background, respectively. The specific domains for binding to $\mathrm{G} \beta \gamma$-subunit complex $(\beta \gamma \mathrm{BD})$, GTP (GTP 1-5), effectors (EBD 1-3) and receptor structures (RBD) were highly conserved in the listed G $\alpha$-subunits of plant and human sources. Putative sites for Nmyristoylation (*), ADP-ribosylation by cholera toxin $(\boldsymbol{\nabla})$ and pertussis toxin $(\nabla)$ are also presented. 
CAT AAC ATG-3' as the forward and 5'-TCA CAA CAG ACC TGC TTC AAA TAG-3' as the reverse primer (1176 mer as the product). In the parallel experiments, $5^{\prime}-\mathrm{TCT}$ ACA ATG AGC TCC GTG TTG C-3' and 5'-TGC CAC GAC CTT AAT CTT CAT GC-3' were used as the primers for the amplification of actin gene fragments as the controls (GenBank accession number AB290348, 725 mer as the product).

\section{RESULTS AND DISCUSSION}

Cloning of Sdga from $\boldsymbol{S}$. dulcis Based on the reported nucleotide sequences of GTP-binding proteins from various biological sources, we isolated a cDNA clone encoding $\alpha$ subunit of heterotrimeric G-protein complex from $S$. dulcis by means of RACE method. The isolated gene, designated Sdga, (GenBank accession number EU489474, 1812 bp) contains an open reading frame of 383 amino acid residues. The primary sequences of the putative amino acids encoded by $S d g a$ showed significant homology with those of $\mathrm{G} \alpha$-proteins from various plant sources such as Arabidopsis thaliana ${ }^{11)}$ and Oryza sativa ${ }^{21}$ (Fig. 1). The five characteristic motifs for GTP-binding and hydrolysis, the unique structures of GTPbinding protein superfamily, ${ }^{10)}$ are well conserved (Fig. 1), and glycine at position 2 , an important lipid modification site for binding of $S d g a$ product to $\mathrm{G} \beta \gamma$-subunit complex, ${ }^{10)}$ was also conserved. The $S d g a$ product contains a domain for receptor-binding (RBD) near the C-terminus and three domains for effector-binding (EBD 1-3) as the characteristic structures of $\mathrm{G} \alpha$-subunits. ${ }^{10)}$ Although a potential ADP-ribosylation site by cholera toxin, arginine residues at position 190 , is conserved in $S d g a$ as well as other $\alpha$-subunits from various biological sources, ${ }^{10,13)}$ the translate of $S d g a$ is lacking in cysteine near the C-terminal, a target of the modification with pertussis toxin observed in several types of animal $\mathrm{G} \alpha$-proteins, Go, Gi, and $\mathrm{Gt}^{10)}$ This structural property of putative ADP-ribosylation sites, presence of cholera toxinbinding site and absence of pertussis toxin-binding site, is also observed in $\mathrm{G} \alpha$-subunits from various plant sources. ${ }^{11,15,21)}$

The deduced amino acid sequence of Sdga translate was further compared with other $\mathrm{G} \alpha$-proteins from various biological sources by molecular phylogenetic tree analysis (Fig. $2 \mathrm{~A})$. It is known ${ }^{9,22)}$ that $>20 \alpha$-subunits of GTP-binding protein have been isolated from human, and they are classified into several subfamilies according to the homology of amino acid sequences and specificity toward effector proteins. In sharp contrast, however, plant $\alpha$-subunits including $S d g a$ appeared very close each other, and only very low divergency was observed in the members of this protein group in plants, although significant differences might occur between monocot and dicot plants (Fig. 2B).

Southern Blot Analysis of $\boldsymbol{S d g a}$ To characterize the genomic organization of $\mathrm{G} \alpha$ gene(s) in $S$. dulcis, Southern hybridization analysis was carried out employing a probe corresponding to the translatable region of Sdga. As shown in Fig. $3 \mathrm{~A}$, the labeled probe hybridized to the restriction digests of genomic DNA, and only one signal was observed in the DNA fragments hydrolyzed with either $X b a \mathrm{I}, X h \mathrm{oI}$, or $E c o$ RI. For further characterization of $\mathrm{G} \alpha$ gene(s) of $S$. dul$c i s$, genomic DNA of the plant was digested with EcoRV of

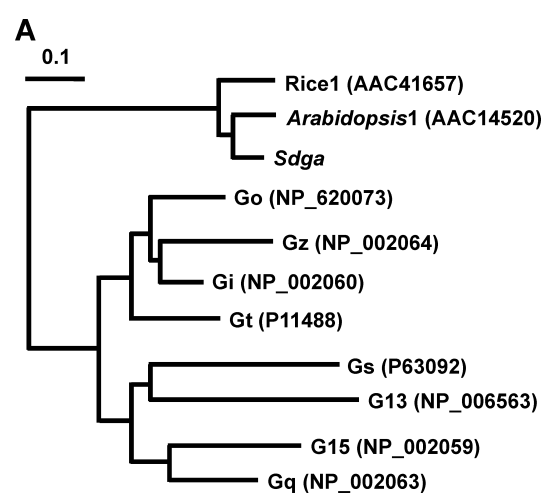

B

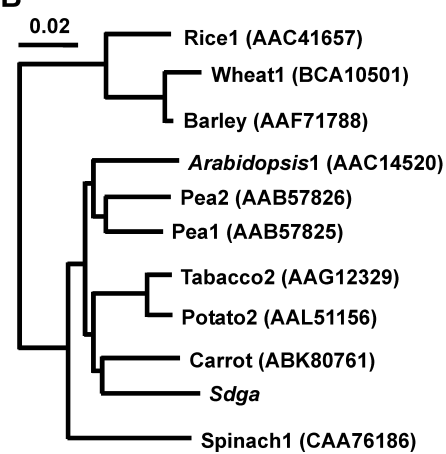

Fig. 2. Phylogenetic Tree Analysis of $\alpha$-Subunits of G-Proteins from Various Biological Sources

Phylogenetic tree for the amino acid sequences of $\mathrm{G} \alpha$-subunits from various biological sources was constructed by NJplot. GenBank accession numbers of the corresponding genes are shown in parentheses. The results obtained for plants and human (A), and for monocot and dicot plants (B) are presented.

which sole restriction site for $S d g a$ locates at the 412 position in its translatable region (Fig. 3B). After probing and visualization reaction, two hybridized signals with comparable intensities were detected (Fig. 3A). These results suggest that $S d g a$ occurs as a sole gene that encodes the $\alpha$-subunit of heterotrimeric GTP-binding protein in $S$. dulcis genome.

Expression of Sdga Gene Tissue-specific expression and possible change in the transcriptional levels of $S d g a$ upon treatment of $S$. dulcis with MJ were examined by RTPCR analysis. Significant expression of $S d g a$ was detected in roots, stems, and leaves, and the transcriptional levels of the gene in these tissues seemed almost comparable (Fig. 4A). Incubation of detached leaves of $S$. dulcis in the presence of MJ appeared not to affect the expression activity of $S d g a$, and it was maintained at almost constant level as far as tested (Fig. 4B). These results imply that heterotrimeric G-protein of $S$. dulcis would participate in MJ-induced activation of diterpenoids biosynthesis, if any, without accompanying notable change in the transcriptional level of Sdga.

Recently, genes encoding the $\alpha$-subunit of heterotrimeric GTP-binding protein complex have been isolated from several plant species, and it has been demonstrated that plant $\mathrm{G} \alpha$ genes are usually organized as one or two copies in the genomes and the products of these genes show only very low diversity. ${ }^{11,21)}$ In sharp contrast, as mentioned above, $>20$ isoforms of $\mathrm{G} \alpha$-subunits function independently in the signal transduction mechanisms in mammalian cells. Therefore the possibility has been proposed ${ }^{12)}$ that plant G-proteins participate into the signaling processes as a non-specific transducer or an enhancer of external stimuli, and activation of specific 
A

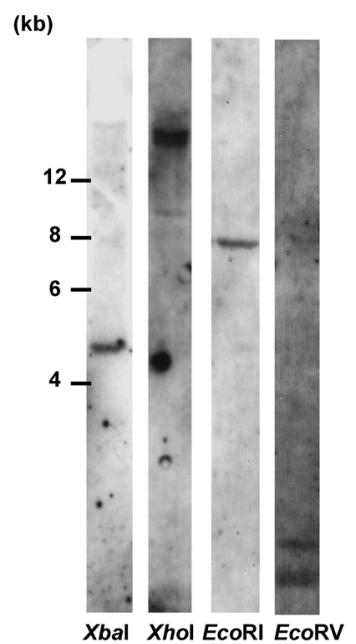

B

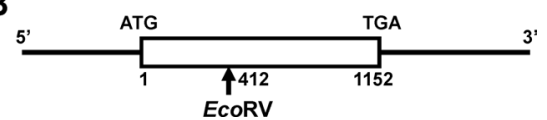

Fig. 3. Genomic Southern Blot Analyses of Sdga

(A) Genomic DNA of $S$. dulcis was digested with XhaI, XhoI, EcoRI, or EcoRV, and, after separation by electrophoresis, the DNA fragments were probed and visualized. (B) Schematic presentation of Sdga and the restriction site. Expected restriction site of EcoRV (412 position of 1152 nucleotides) in the coding region (presented as open column) of the gene is indicated by an arrow.

\section{A}

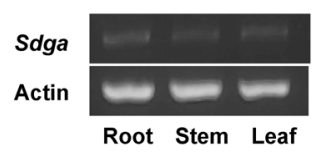

B

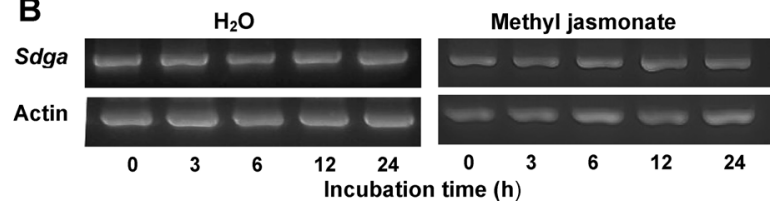

Fig. 4. RT-PCR Analyses of the Expression Levels of Sdga

(A) Total RNA was prepared from roots, stems, and leaves of $S$. dulcis, and after the RT reaction, PCR was performed employing the appropriate primer pairs described in the text. The DNA fragment of actin gene of $S$. dulcis was also amplified as control. (B) Changes in expression levels of Sdga were examined after incubation of the leaf tissues with MJ. The MJ-treated leaves were harvested at regular intervals, and the RNA was isolated from the samples. Control treatment received $\mathrm{H}_{2} \mathrm{O}$ instead of MJ.

CAM might be, in part, a trigger event that leads plant cells to appropriate cellular responses. In the present study, it has been demonstrated that, although $S$. dulcis is a unique plant with only one CAM gene, the $\alpha$-subunit of heterotrimeric Gprotein complex of this plant is also encoded by a sole gene. How does $S$. dulcis respond to various signals with only one $\mathrm{G} \alpha$-subunit and one CAM gene? Certain functional proteins located downstream of the signaling cascade, such as small
GTP-binding proteins and protein kinases, might play specific roles to evoke appropriate cellular responses toward various stimuli. Alternatively, it might be also possible that several isoforms of these two proteins with different functions could be synthesized by post-translational modifications from the gene products. It has been recently reported that heterotrimeric G-protein $\gamma$-subunits provide functional selectivity in signaling processes in $A$. thaliana including $\mathrm{MJ}$ induced defense responses. ${ }^{5}$ However, the $\gamma$-subunit of $A$. thaliana is also encoded by only two genes, and therefore, it seems that $\mathrm{G} \gamma$-subunits themselves might not be the key determinant of the variety of cellular responses mediated by heterotrimer G-protein complex against numerous signals. Further studies are in progress in our laboratory to elucidate the physiological functions of the components of GTP-binding protein complex in jasmonates-induced responses in $S$. dulcis.

Acknowledgement This work was supported in part by a Grant-in-Aid from the Ministry of Education, Culture, Sports, Science and Technology of Japan.

\section{REFERENCES}

1) Turner J. G., Ellis C., Devoto A., Plant Cell, 14, S153-S164 (2002).

2) Weiler E., Naturwissenschaften, 84, 340-349 (1997).

3) Wasternack C., Ann. Bot., 100, 681-697 (2007).

4) Fits L., Memelink J., Science, 289, 295-297 (2000).

5) Trusov Y., Rookes J. E., Chakravorty D., Armour D., Schenk P. M., Botella J. R., Plant Physiol., 140, 210-220 (2006).

6) Barnes S. A., McGrath R. B., Chua N. H., Trends Cell Biol., 7, 21-26 (1997).

7) Ritche S., Gilroy S., Plant Physiol., 124, 693-702 (2000).

8) Bischoff F., Molendijk A., Rajendrakumar C. S., Palme K., Cell. Mol. Life Sci., 55, 233-256 (1999).

9) Gilman A. G., Annu. Rev. Biochem., 56, 615-649 (1987).

10) Simon M. I., Strathmann M. P., Gautam N., Science, 252, 802-808 (1991).

11) Ma H., Yanofsky M. F., Meyerowitz E. M., Proc. Natl. Acad. Sci. U.S.A., 87, 3821-3825 (1990).

12) Ellis B. E., Miles G. P., Science, 292, 2022-2023 (2001).

13) Hossain M. S., Koba T., Harada K., Genes Gene. Syst., 78, 127-138 (2003).

14) Assmann S. M., Science, 310, 71-73 (2005).

15) Asakura Y., Kurosaki F., Biol. Pharm. Bull., 30, 1800-1804 (2007).

16) Hayashi T., Okamura K., Kawasaki M., Morita N., Phytochemistry, 33, 353 -356 (1993).

17) Kasidimoko N. K., Lee J. B., Hayashi T., Chem. Pharm. Bull., 53, $780-782$ (2005).

18) Yamakawa H., Mitsuhara I., Ito N., Seo S., Kamada H., Ohashi Y., Eur. J. Biochem., 268, 3916-3929 (2001).

19) Ishigaki E., Sugiyama R., Kurosaki F., Biol. Pharm. Bull., 28, 11091112 (2005).

20) Saitoh D., Asakura Y., Kasidimoko N. K., Shite M., Sugiyama R., Lee J. B., Hayashi T., Kurosaki F., Biol. Pharm. Bull., 30, 1161-1163 (2007).

21) Seo H. S., Kim H. Y., Jeong J. Y., Lee S. Y., Cho M. J., Bahk J. D., Plant Mol. Biol., 27, 1119-1131 (1995).

22) Neer E. J., Cell, 80, 249-257 (1995). 